\title{
Editorial
}

\section{Vision of Journal of Cardiac Critical Care}

\author{
Poonam Malhotra Kapoor ${ }^{1}$ \\ 1 Department of Cardiac Anesthesia, Cardiothoracic Center, All India \\ Institutes of Medical Sciences, New Delhi, India \\ J Card Crit Care TSS 2017;1:1-2.
}

It is a great privilege to introduce the Journal of Cardiac Critical Care, a new international publication and a multidisciplinary journal, aiming to promote the dissemination of scientific information on the treatment of critically ill cardiac patients and publish high quality scientific papers covering different aspects of those patients. This journal will cover a broad spectrum of therapeutic disciplines, such as cardiac surgery, emergency medicine, perioperative cardiac anesthesia care, cardiology, internal medicine, pediatrics, infectious diseases, organ transplantation, neurology, pulmonary medicine, and pain management in cardiac adult and pediatric patients.

\section{Cardiac Critical Care: An Emerging Technology}

Cardiac critical care medicine is a rapidly expanding field. Recent progress in medical science and technology has led to a significant increase in the number of patients with lifethreatening conditions that can benefit from increasingly wide-ranging monitoring and care, especially in the cardiac critical care intensive care unit (ICU). Significant resources are currently invested in the complex effort to discover and develop new approaches dedicated to improve the progress of these patients and to ensure a fast clinical and functional recovery $^{1}$ (-Fig. $\mathbf{1}$ ).

The emerging technology has been overlapped in different fields such as echocardiography, use of extracorporeal membrane devices, ventricular assist devices, hemodynamic monitoring, and pain and sepsis management in the ICU. The merger of so many diverse disciplines has required a vision of forward thinking and innovation, thus replacing the conventional way of management of critical care patient to achieve new dimensions in the field. Cardiac critical care is thus a convergence of different specialties brought together by overlapping emerging technologies, imaging, and diagnostics being the chief common point. ${ }^{2}$
This niche field of medicine is undergoing changes in teaching, training, research, board examinations, and credentialed specifications. This new branch is here to stay and achieve rapid growth. The leaders will be from many specialties and associations, coming together under the same super specialty. Unity in diversity for welfare of a common source will be the common agenda. ${ }^{3}$

\section{Bundles and Protocols Applied in Cardiac Critical Intensive Care Unit}

Several internationally accepted and recommended bundles, protocols, and guidelines will be incorporated in this journal on various subjects pertaining to critical care. This is a work in progress, and more standardized practices are continuously being introduced and applied on the sick cardiac patient.

One of the main goals of any medical journal is to contribute to the evidence base necessary to improve medical practice. ${ }^{4}$ As a measure of social responsibility, the Journal of Cardiac Critical Care will stimulate publication of research that could be easily translated into current practice, and in so doing will contribute to improved outcomes for critically ill patients. Undoubtedly, achieving these improvements represents an extremely daunting task. It entails not only a complex and multidisciplinary approach but also a high level of expertise in various different medical specialties. Covering different topics of medicine, ranging from preclinical to clinical studies and having the objective to publish the most recent advances in the field of research related to cardiac critical care, the journal will promote the most upto-date understanding of different pathophysiological mechanisms involved in the progression of disease, together with the most contemporary accepted treatment of critically ill patients.

The authors invite clinicians, researchers, and students to publish relevant papers in the Journal of Cardiac Critical Care, to enjoy together the growth of the journal, and to share with

\footnotetext{
Address for correspondence Poonam Malhotra Kapoor, Department of Cardiac Anesthesia, Cardiothoracic Center, All India Institutes of Medical Sciences, Ansari Nagar, New Delhi, Delhi 110029, India (e-mail: drpoonamaiims@gmail. com).
}

DOI https://doi.org/ 10.1055/s-0037-1607338.
Copyright (c) 2017 Official Publication of License terms The Simulation Society (TSS), accredited by International Society of Cardiovascular Ultrasound (ISCU) 


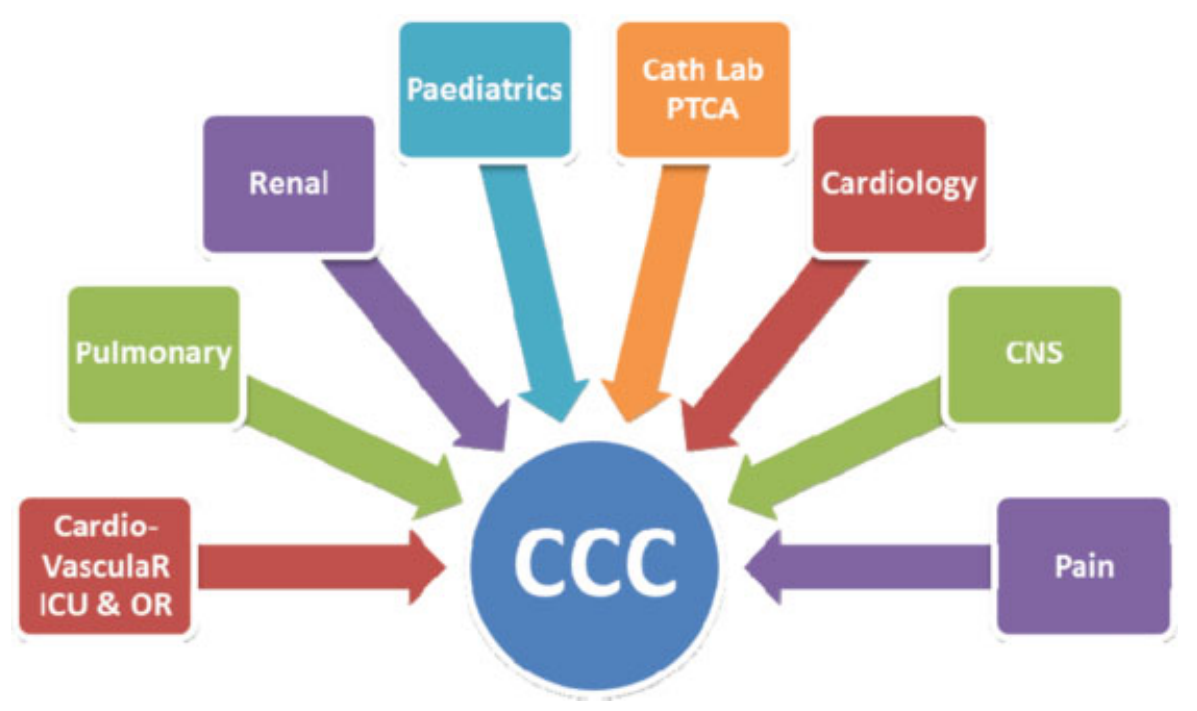

Fig. 1 Venn diagram depicting multiple organ failure typical of a critically ill patient in cardiac critical care. CNS, central nervous system; ICU, intensive care unit; OR, operating room; PTCA, percutaneous transluminal coronary angioplasty.

the authors the success of this enthusiastic and visionary academic initiative.

The Journal of Cardiac Critical Care will offer a unique integrative approach by combining the expertise of the different subspecialties of cardiology, emergency, and critical care and intensive care medicine in the management of patients with cardiac critical disease. Reading through the journal, all health care professionals working in this field will be offered a continuous update that may help them to improve the diagnosis, treatment, and outcome of patients with cardiovascular diseases that are under their care. The journal will have a strong educational focus on continuing progress in the medical field. In addition to original research papers and editorials, ample space will be devoted to educational materials: recommendation papers by scientific bodies of different subjects, invited reviews, illustrative clinical cases, practical tips and tricks, how-to-do articles, and video commentaries on special subjects.

Conditions pertaining to patient's quality of life after ICU admission such as sepsis, brain studies for delirium, and alternative ventilator support for selected patients in ICU are going to be added focus content of the journal. ${ }^{5}$ The authors endeavor to extend authorship and readership benefits to people under the Education and Fellowship courses under its banner in cardiac critical care ICU so that contributors are maximized and globally distributed.

\section{Peer Review of Articles Is the Backbone}

The journal will accept submissions dealing with all aspects of cardiac critical care. Articles will undergo a thorough peer review by a group of editors, statistical consultants, and reviewers representing the full scientific expertise of the cardiac critical care. The editorial team will process submissions within a very short time, and accepted papers will be posted online on the Web site of the journal within 8 weeks after acceptance. Citations on PubMed Central will be obtained as quickly as possible.

The editorial team hopes to provide the readership of Journal of Cardiac Critical Care a very attractive information source of great practical utility in daily clinical practice that progressively will become an established body of reference and guidance in the management of cardiovascular syndromes.

The cardiac critical care division worldwide is still in its infancy, but the journal has taken up the challenges of developing this evolving specialty with the entire modern infrastructure at its disposal for state-of-the-art delivery of health care to the most critically ill adult and pediatric cardiac patients. Long live the JCCC!

\section{References}

1 Munro CL, Savel RH. Stepping forward in practice through research. Am J Crit Care 2014;23(01):4-6

2 Vrints CJ. Welcome to the European heart journal: acute cardiovascular care. Eur Heart J Acute Cardiovasc Care 2012;1(01): 3-4

3 Hussain A, Mustafa N, Al Ali R, Almodaimegh H. Adult cardiac critical care at the King Abdulaziz Cardiac Center. Eur Heart J Suppl 2014;16:B52-B54

4 Grief RT. Born from the ashes to blossom: a short insight into the development of TACC. Trends AnaesthCrit Care 2015;5:49

5 Lumb PD. Impact factor. Editorial. J Crit Care 2010;25(03):365-366 